Si ngl e fi el d- of-vi ew tomgr aphi c i maging of 3D i mpur i ty emi ssi on di st ri but i on i n magnet i zed edge pl astra of LHD

\begin{tabular}{|l|l|}
\hline $\begin{array}{l}\text { jour nal or } \\
\text { publ i cat i on } \mathrm{ti} \text { t l e }\end{array}$ & Revi ew of Sci ent i f i c I nst r ument s \\
\hline vol une & 89 \\
\hline number & 12 \\
\hline page $\mathrm{r}$ ange & 123502 \\
\hline year & $2018-12-10$ \\
\hline URL & ht t p: //hdl . handl e. net /10655/00012888 \\
\hline
\end{tabular}




\title{
Single field-of-view tomographic imaging of 3D impurity emission distribution in magnetized edge plasma of LHD
}

\author{
Taisuke KOBAYASHI ${ }^{1)}$, Masahiro KOBAYASHI ${ }^{1)}{ }^{2)}$, Naofumi IWAMA ${ }^{2)}$, Arseniy $\mathrm{KUZMIN}^{2)}$, \\ Motoshi GOTO ${ }^{1,2)}$, Gakushi KAWAMURA ${ }^{1,2)}$, and the LHD Experimental Group ${ }^{2)}$ \\ 1) SOKENDAI (The Graduate University for Advanced Studies), 322-6 Oroshi-cho, Toki 509-5292, Japan \\ 2) National Institute for Fusion Science, 322-6 Oroshi-cho, Toki 509-5292, Japan
}

\section{Introduction}

In magnetically confined fusion reactors, understanding of impurity transport in edge region is a critical issue to control impurity radiation in divertor region as well as impurity influx into the confinement region. Because of the very fast transport of charged particles parallel to magnetic field lines, the resulting impurity distributions are largely affected by magnetic field structure [1].

LHD (Large Helical Device) is a heliotron type device, in which magnetic field configuration is inherently non-axisymmetric [2]. The edge region is called stochastic magnetic layer, where magnetic islands with different mode numbers overlap each other to induce chaotic field line trajectories. In this region, strong poloidal and toroidal asymmetry of impurity emission is predicted by plasma transport simulations [ 3, 4]. Two-dimensional (2D) emission distribution measurements have been conducted so far with spectrometers in edge region of LHD in order to study relation between plasma transport and magnetic field structure [5,6,7]. However, the detailed comparison between the measured impurity emission and magnetic field structure has been limited because of the line integration effect along the line of sight (LOS).

In this paper, we propose a new tomographic scheme to reconstruct three dimensional (3D) impurity emission distribution from single field of view 2D measurement in magnetized plasma by redistributing the signal along the line of sight. For this purpose, we utilize a characteristic of the magnetized plasma, where plasma transport parallel to the magnetic field line is much larger than those perpendicular to magnetic field lines. The scheme is useful not only for helical devices with the complex magnetic field structure, but also for tokamaks in case of limited number of observation ports. The paper is organized as follows. In section 2, the experimental set up of the spectroscopy system as well as magnetic field structure of LHD are explained. In section 3, the tomographic scheme is described with various regularization methods tested and its feasibility is discussed. The scheme is applied to experimental data in section 4 . The paper is summarized in section 5 .

\section{Experimental setup}

The relation between the viewing area of the spectrometer and the LHD torus is shown in Fig.1. The open field lines in the edge region go around torus along helical coils until they are cut by the divertor plates. The trajectories of the fields lines are also shown in Fig.1 with red and yellow colors. The spectrometer views the plasma from the outboard side. The line of sight (LOS) is inclined toward top of torus and toward toroidal direction such that the LOS is almost tangential to the edge magnetic field lines. Figure 2 shows the field of view of the spectrometer, where 131 optical fibers are distributed to spatially resolve the edge region. The viewing area covers the last closed flux surface (LCFS), divertor legs, X-point, and divertor plates. The groups of red and yellow lines in Fig. 2 indicate divertor leg field lines, each group connects to the different divertor plate arrays, respectively.

At the entrance slit of the spectrometer, the optical fibers are vertically aligned with separation of $150 \mu \mathrm{m}$ each other. The image of the fibers is focused at the exit slit, where a charge couple device (CCD) camera is equipped. In order to obtain good focus of the fiber image in wide area on the CCD detector, an astigmatism is suppressed by introducing toroidal and spherical mirrors in the spectrometer. The detail of the optics of the spectroscopy system is described in ref.[ 8 ]. The spectrometer can change diffraction gratings remotely between 150, 300, and 2400 grooves $/ \mathrm{mm}$. In the present measurements, the grating of 150 grooves $/ \mathrm{mm}$ is used for measurements of CII $\left(1 \mathrm{~s}^{2} 2 \mathrm{~s} 2 \mathrm{p} 3 \mathrm{~s}^{4} \mathrm{P}^{\mathrm{o}}-1 \mathrm{~s}^{2} 2 \mathrm{~s} 2 \mathrm{p} 3 \mathrm{p}{ }^{4} \mathrm{P}, 514 \mathrm{~nm}\right)$, and the grating of 2400 grooves/mm is used for CIII $\left(1 \mathrm{~s}^{2} 2 \mathrm{~s} 3 \mathrm{~s}\right.$ $\left.{ }^{3} \mathrm{~S}-1 \mathrm{~s}^{2} 2 \mathrm{~s} 3 \mathrm{p}{ }^{3} \mathrm{P}^{\mathrm{o}}, 465 \mathrm{~nm}\right)$ and $\mathrm{CIV}\left(1 \mathrm{~s}^{2} 5 \mathrm{f}^{2} \mathrm{~F}^{\mathrm{o}}-1 \mathrm{~s}^{2} 6 \mathrm{~g}{ }^{2} \mathrm{G}, 1 \mathrm{~s}^{2} 5 \mathrm{~g}\right.$ ${ }^{2} \mathrm{G}-1 \mathrm{~s}^{2} 6 \mathrm{~h}^{2} \mathrm{H}^{\mathrm{o}}$, $\left.466 \mathrm{~nm}\right)$. The wavelength resolutions near the center of the CCD are $1.07 \mathrm{~nm}$ and $0.06 \mathrm{~nm}$ with the gratings of 150 and 2400 grooves/mm, respectively. Exposure time and cycle time are set to $0.09-0.19 \mathrm{sec}$ and $0.2-0.3 \mathrm{sec}$, respectively. These settings were changed depending on the gratings and the plasma parameters.

\section{Tomography scheme}

\section{3-1. Field-line aligned 3D grid generation}

For the present tomography scheme, we make use of the plasma transport feature that the transport is much 
faster along magnetic field lines as compared to the transport perpendicular to the field lines, which leads to smooth distribution of physical quantity along the magnetic field lines. In order to incorporate this effect in the projection matrix and in the regularizations of the tomographic inversion, the voxellation of the measurement volume is made such that the vertices of voxels are aligned along magnetic field lines.

Figure 3 shows 2D distributions of magnetic field line connection length $\left(L_{c}\right)$ at different cross sections perpendicular to the LOS. $L_{C}$ is defined as a length of field lines trajectory between divertor plates. The length labels in each panel in Fig.3 (also later figures 4, 5 and 10) show the distance from the observation point to the cross section, $L_{\mathrm{dp}}$. The region with red color represents $L_{c}$ more than $10^{4}$ $\mathrm{m}$, which corresponds to a confinement region. Around the confinement region, the so-called stochastic magnetic field layer is formed, where regions with different $L c$ 's (yellow, green and blue) are mixed. $L_{c}$ rapidly decreases in the radially outward direction as seen in the change of the color in the figure. Two divertor legs are visible with thin lines of blue color, and they cross each other at X-point (The color of the divertor legs in Figs.1 and 2 are not relevant to the $L_{c}$, but only for visualization.).

The preparation of voxels for projection matrix has been done taking into account these magnetic field structure. The grid starts from the region just inside of LCFS and extends radially outward along the divertor legs, as shown in Fig.4. This selection of grid area is based on possible emission area of CII, CIII, CIV, whose ionization potentials are 24.4, 48.0 and $64.7 \mathrm{eV}$, respectively, so that they rarely emit inside LCFS, where the temperature is usually higher than a few hundred $\mathrm{eV}$. It is also considered that in the deep blue region in Fig.3, where the $L_{\mathrm{C}}$ is less than $5 \mathrm{~m}$, the plasma density is so low that impurity emission (except for neutrals) is very weak. The gird is first constructed at $4.0 \mathrm{~m}$ from the observation point. Then each vertex of the gird is traced along magnetic field lines to construct 3D grid structure. Flux tube cross-section in edge region is strongly deformed along magnetic field due to magnetic shear. Therefore, the grid was carefully constructed not to be crushed during the tracing, taking into account the magnetic field structure. At first, the very fine grid (typical size of $51 \mathrm{~mm}$, and minimum size of 1.3 $\mathrm{mm}$ ) was constructed to accommodate the strong deformation. Then the neighboring voxels with similar $L_{\mathrm{C}}$ are unified in order to reduce the number of voxels as much as needed to remedy the ill condition in tomographic inversion. Eventually, in the present analysis, 1464 voxels in the entire measurement volume, which consists of 242 flux tubes, are constructed successfully, as shown in Fig. 4. The resulting spatial resolution of the grid along the line of sight is $30 \mathrm{~cm}$. With this non-uniform voxellation, the elements $L_{\mathrm{m}, \mathrm{k}}$ of the projection matrix $\boldsymbol{L}$ in image analysis are evaluated as

$$
L_{\mathrm{m}, \mathrm{k}}=V_{\mathrm{m}, \mathrm{k}} / A_{\mathrm{m}, \mathrm{l}},
$$

where $m$ and $k$ are the indices of the optical fibers, and the voxels in the measurement domain, respectively. $l$ is the index of each cross section perpendicular to the line of sight. Then, $V_{\mathrm{m}, \mathrm{k}}$ is the $k$-th voxel volume in the field of view of the $m$-th fiber, and $A_{\mathrm{m}, \mathrm{l}}$ is the area in the $l$-th cross section covered by the field of view of the $m$-th fiber.

\section{3-2. Regularization operators and solution}

Using the provided $\boldsymbol{L}$, an unknown vector $\boldsymbol{E}$ which represents the spatial distribution of emissivity in measurement volume is related to a data vector $\boldsymbol{S}$ which represents the observed image at detector, as follows:

$$
\boldsymbol{L E}=\boldsymbol{S} .
$$

The size of $\boldsymbol{E}$ is the number of voxels $\mathrm{K}=1464$ while the size of $\boldsymbol{S}$ is the number of fibers $\mathrm{M}=131 . L_{\mathrm{m}, \mathrm{k}}$ in Eq. (1) is the contribution rate of the voxel value $E_{\mathrm{k}}$ to the output $S_{\mathrm{m}}$. With the under-determined equation (2) $(K>M)$, the inverse problem to obtain $\boldsymbol{E}$ from $\boldsymbol{S}$ will be ill-conditioned as is often the case in plasma imaging and especially in our experiment with single field-of-view. In order to obtain a good solution, one needs an appropriate regularization.

In least-squares approach, a well-known strategy is to solve the problem in the scheme of minimization under constraint, which is reduced to minimizing Lagrangian functions of the form

$$
\Lambda(E)=\frac{1}{M}\|L E-S\|^{2}+\text { regularization terms. }
$$

A variety of regularization terms that lead to linear and nonlinear solutions have been investigated in plasma imaging, which is usually given a strong limitation in the number of fields of view. When applied to plasma imaging, a notable feature is to use derivative operators, which are effective for smooth plasma profiles. One finds typical works in the L2-norm regularizations of Tikhonov type [9$13]$ and a modified type [14, 15] and also, in nonlinear regularizations of Fisher type [16-19] and Hopfield neural net [20]. In Ref. 18, an anisotropic derivative operator was contrived and examined for 2D tomography in JET.

In the present analysis, within the Tikhonov scheme, we introduce a new anisotropic regularization, taking into account the transport feature of the magnetized plasma which should have distribution smoothed much along flux tubes as described in section 3-1. That is, we define $\Lambda(\boldsymbol{E})$ as

$$
\Lambda(E)=\frac{1}{M}\|L E-S\|^{2}+\gamma_{1}\left\|D_{1} E\right\|^{2}+\gamma_{2}\left\|D_{2} E\right\|^{2}+\gamma_{3}\|T E\|^{2}(3) .
$$

The mean of squared residuals is assisted with three terms. The first term is the most important for regularization and 
possesses the $2^{\text {nd }}$-order derivative operator $\mathbf{D}_{1}$ that is defined over three neighboring voxels along each flux tube. The next term is introduced, in imaging, to control spatial roughness perpendicular to the field lines and possesses the $1^{\text {st }}$-order derivative operator $\mathbf{D}_{2}$, which is defined for geometrical facility between two neighboring flux tubes. The last term is the proper term of Tikhonov regularization with a diagonal matrix $\mathbf{T}$, which is originally the identity matrix I. The multipliers $\gamma_{1}, \gamma_{2}$ and $\gamma_{3}$ are positivevalued and used as parameters for weighting three terms.

With the extended Lagrangian function, we employ the Cholesky decomposition for numerically reliable minimization. The $\Lambda(\boldsymbol{E})$ is minimized with respect to $\boldsymbol{E}$ by solving the following linear equation, which has a unique solution:

$\left\{\boldsymbol{L}^{\mathrm{T}} \boldsymbol{L}+M \gamma_{1}\left(\boldsymbol{D}_{1}{ }^{\mathrm{T}} \boldsymbol{D}_{1}+\alpha \boldsymbol{D}_{2}{ }^{T} \boldsymbol{D}_{2}+\beta \mathbf{T}^{\mathrm{T}} \mathbf{T}\right)\right\}=\boldsymbol{L}^{T} \boldsymbol{S}$

with $\alpha=\gamma_{2} / \gamma_{1}$ and $\beta=\gamma_{3} / \gamma_{1}$. Since the matrix $\mathbf{P}=$ $\boldsymbol{D}_{1}{ }^{T} \boldsymbol{D}_{1}+\alpha \boldsymbol{D}_{2}{ }^{T} \boldsymbol{D}_{2}+\beta \mathbf{T}^{\mathrm{T}} \mathbf{T}$ is symmetric and positive definite, the Cholesky decomposition $\mathbf{P}=\mathbf{R}^{\mathrm{T}} \mathbf{R}$ with an upper triangular matrix $\mathbf{R}$ is possible. Then, we have an equation of conventional form: $\left(\boldsymbol{L}^{T} \boldsymbol{L}+\mathrm{M} \gamma_{1} \mathbf{R}^{\mathrm{T}} \mathbf{R}\right) \boldsymbol{E}=$ $\boldsymbol{L}^{T} \boldsymbol{S}$. With this rewriting, the singular value decomposition (SVD) $\boldsymbol{L} \mathbf{R}^{-1}=\mathbf{U} \boldsymbol{\Sigma} \mathbf{V}^{\mathrm{T}}$ leads the solution $\boldsymbol{E}$ to the ordinary form of series expansion. That is, we have

$$
E\left(\gamma_{1}\right)=\sum_{m=1}^{M} w_{m}\left(\gamma_{1}\right) \frac{\left(S, \mathbf{u}_{m}\right)}{\sigma_{m}}\left(\mathbf{R}^{-1} \mathbf{v}_{m}\right),
$$

with the Tikhonov window

$$
\mathbf{w}_{\mathbf{m}}\left(\gamma_{1}\right)=\frac{1}{1+M \gamma_{1} / \sigma_{m}^{2}}
$$

With the orthonormal column vectors $\mathbf{u}_{\mathrm{m}}$ and $\mathbf{v}_{\mathrm{m}}$ of the matrices $\mathbf{U}$ and $\mathbf{V}$, one has a basis system $\mathbf{R}^{-1} \mathbf{v}_{\mathrm{m}}$ for $\boldsymbol{E}\left(\gamma_{1}\right)$ and using the series $\mathbf{u}_{\mathrm{m}}$ as the basis system for $\boldsymbol{S}$. With the singular values $\sigma_{\mathrm{m}}$ (diagonal elements of $\boldsymbol{\Sigma}$ ) in descending order, one has the monotonically decreasing function $\mathrm{w}_{\mathrm{m}}\left(\gamma_{1}\right)$ for tapering down the expansion coefficients $\left(\boldsymbol{S}, \mathbf{u}_{\mathrm{m}}\right) / \boldsymbol{\sigma}_{\mathrm{m}}$, which may increase in the region of large $m$ (high spatial-frequency); (a, b) denotes the inner product of vectors $\mathbf{a}$ and $\mathbf{b}$. It should be noted that the inverse of the triangular matrix, $\mathbf{R}^{-1}$, is well-conditioned in usual and calculated numerically with high reliability.

With respect to the solution $\boldsymbol{E}\left(\gamma_{1}\right)$, the generalized cross-validation (GCV) for optimizing the value of $\gamma_{1}$ is given as

$$
\operatorname{GCV}\left(\gamma_{1}\right)=\frac{\varepsilon^{2}}{\left(1-M^{-1} \sum_{m=1}^{M} w_{m}\left(\gamma_{1}\right)\right)^{2}},
$$

where $\varepsilon^{2}=\mathrm{M}^{-1}\left\|\boldsymbol{L} \boldsymbol{E}\left(\gamma_{1}\right)-\boldsymbol{S}\right\|^{2}$ is the attained mean square error (MSE). Since the matrix $\mathbf{R}$ and thus all the SVD components involve the weights $\alpha$ and $\beta$ as parameters, the criterion of minimum GCV is valid for particular values of $\alpha$ and $\beta$.

The term $\left\|\boldsymbol{D}_{1} \boldsymbol{E}\right\|^{2}$ is defined with the central difference between the second-order differentials along flux tube. As a boundary condition at the both edges of the field lines in the measurement volume, $\nabla_{\|}=0$ and $\nabla_{\|}^{2}=$ 0 have been tested with respect to the derivative $\nabla_{\|}$along field lines. It is found that solutions are almost independent of such boundary conditions. Therefore, in the present analysis, we used the boundary condition $\nabla_{\|}=0$ for $\left\|\boldsymbol{D}_{1} \boldsymbol{E}\right\|^{2}$. The term $\left\|\boldsymbol{D}_{2} \boldsymbol{E}\right\|^{2}$ is defined with the one-sided difference between the neighboring voxels perpendicular to field lines. The differentiation is introduced only for the voxels having similar $L_{\mathrm{C}}$ values and thereby, for derivatives along the poloidal direction and along divertor legs as seen in the $L_{\mathrm{C}}$ distributions in Fig.4. This treatment is based on the plasma transport feature in the magnetic field as explained above.

\section{3-3 Algorithm of image reconstruction}

There are two reasons for adding the term $\|\mathbf{T E}\|^{2}$ in Eq. (3). Firstly, despite of the success in Refs. 9-12, the derivative operators like the Laplacian operator are never well-conditioned in general. In the present scheme of single field-of-view tomography, it is found that the inverse matrix $\mathbf{R}^{-1}$ cannot be calculated without the original Tikhonov term $\|\boldsymbol{E}\|^{2}$. Secondarily, the diagonal matrix $\mathbf{T}$ is practically used for applying the constraint of non-negative valued emissivity. That is, when the initial solution $\boldsymbol{E}\left(\gamma_{1}\right)$ with the identity matrix $\mathbf{T}=\mathbf{I}$ has negative values at some voxels, we anticipate that the voxels have very low emissivity and therefore, we set the corresponding diagonal elements $T_{k, k}$ of $\mathbf{T}$ to be large positive values as additional penalties. Then, the voxel values are forced to approach zero in the next iterative step of minimizing the Lagrangian function. With the matrix $\mathbf{T}$ so-updated, $\mathbf{R}$ is recalculated at each iteration. Experimentally, it is found that in most of the cases the iteration converges and a reasonable non-negative solution is obtained.

After all, the iterative algorithm of image reconstruction is as follows:

[Step 1] Set the values of $\alpha$ and $\beta$.

[Step 2] $\mathbf{T}^{(0)}=\mathbf{I}$

[Step 3] For $\mathbf{T}^{(\mathrm{n})}$, obtain $\mathbf{R}^{(\mathrm{n})}, \mathbf{L} \mathbf{R}^{-1(\mathrm{n})}$ and the SVD.

[Step 4] Find $\gamma_{1}$ that minimizes $\operatorname{GCV}\left(\gamma_{1}\right)$.

[Step 5] For the minimizer $\gamma_{1}$, calculate $\boldsymbol{E}\left(\gamma_{1}\right)$ according to Eq. (5).

[Step 6] For all $k$ where we have $E_{\mathrm{k}}<0$, set $\mathrm{T}_{\mathrm{k}, \mathrm{k}}{ }^{(\mathrm{n}+1)}$ to a large value, for example, $10^{7}$. Go to Step 3 for $\mathrm{n}+1$.

The procedure from Step 3 to Step 6 is repeated iteratively until we have no negative-valued new voxels 
anywhere. With this iteration, the whole procedure starting with Step 1 is repeated in order to find good values of $\alpha$ and $\beta$, especially, the value of $\alpha$. The software is interactive only for the choice of $\alpha$ and $\beta$.

\section{3-4 Numerical simulations}

\section{[1] Optimization of regularization parameters}

To check the feasibility of tomographic imaging, numerical tests have been conducted. Figure 5 shows one of the tested phantoms $\boldsymbol{E}_{\mathrm{ph}}$, which imitates that the emissivity is distributed along poloidal direction and divertor legs. The emission is nonuniform along flux tubes such that the intensity increased linearly with the distance from the observation point, $L_{\mathrm{ph}}$. In calculation, $1 \%$ Gaussian noise with zero mean is added to the projection $\boldsymbol{L E}_{\mathrm{ph}}$ for producing the data $\boldsymbol{S}$, and $\gamma_{1}$ is scanned in a wide range from $10^{-11}$ to $10^{4}$ for fixed values of $\alpha$ and $\beta$. Figure 6 shows an obtained behavior of the GCV, the MSE $\varepsilon^{2}$ and the reconstruction error $\delta$ as functions of $\gamma_{1}$, for a particular series of Gaussian random number. Corresponding to the non-uniform voxellation, the error $\delta$ is defined as

$$
\delta=\frac{\sum_{\mathrm{k}=1}^{\mathrm{K}}\left|\mathrm{E}_{\mathrm{ph}, \mathrm{k}}-\mathrm{E}_{\mathrm{k}}\left(\gamma_{1}\right)\right| \mathrm{V}^{\mathrm{k}}}{\sum_{\mathrm{k}=1}^{\mathrm{K}} \mathrm{V}^{\mathrm{k}}} .
$$

Here $V^{\mathrm{k}}$ is the own volume of the $k$-th voxel and has a relation $V^{\mathrm{k}} \geq \sum_{\mathrm{m}=1}^{\mathrm{M}} V_{\mathrm{m}, \mathrm{k}}$ with the volumes $V_{\mathrm{m}, \mathrm{k}}$ which are defined in Eq. (1). Each voxel is not completely contained in the measurement area of fibers and further, there are spaces among fibers as seen in Fig.2. The error $\delta$ is a quantitative estimate of the deviation of the obtained $\boldsymbol{E}\left(\gamma_{1}\right)$ from the assumed $\boldsymbol{E}_{\text {ph }}$ over the entire 3D measurement volume. With the decrease of $\gamma_{1}$, the regularization along flux tubes is weakened by the effect of Tikhonov window $\mathrm{w}_{\mathrm{m}}\left(\gamma_{1}\right)$. As a result, the recovered projection $\boldsymbol{L} \boldsymbol{E}\left(\gamma_{1}\right)$ tends to match precisely the projection data $\boldsymbol{S}$. The ceiling of $\varepsilon^{2}$ at $\left\|\boldsymbol{E}_{\text {ph }}\right\|^{2}$ for very large $\gamma_{1}$ and the subsequent monotonic decrease of $\varepsilon^{2}$ seen in Fig. 6 give a proof of correct calculation. On the other hand, the GCV has a minimum around $\gamma_{1}=10^{-5}$, below which the GCV gradually separates from the value of $\varepsilon^{2}$. The increase of GCV for smaller $\gamma_{1}$, which suggests that the denominator of GCV decreases faster than the numerator, corresponds to the appearance of too fine structure in reconstruction due to too weak regularization. As expected, the minimum of GCV agrees well with the minimum of the reconstruction error.

This result of reconstruction in Fig. 6 has been obtained for $\alpha=10^{-2}$ and $\beta=10^{-6}$. When the test is made in the range of $10^{-5} \leq \alpha \leq 10^{-1}$ and $10^{-6} \leq \beta \leq 10^{-2}$, the $\boldsymbol{E}\left(\gamma_{1}\right)$ obtained with the criterion of minimum GCV is not changed significantly by the value of $\beta$. Therefore, $\beta$ can be fixed at a small value of the level indispensable for sure calculation of $\mathbf{R}^{-1}$. However, the change of $\alpha$ gives a significant change of reconstruction. In regarding that the minimum of GCV for a set of $\left(\gamma_{1}, \alpha\right)$ looks like indicating the minimum of $\delta$ in many tests, the value of $\alpha$ is selected as such without support of statistical mathematics, $\alpha=10^{-2}$ in this example. Recalling that these values of $\alpha$ and $\beta$ are the weights in ratio among three regularization terms in Eq. (3), we note that the very small value $\beta=10^{-6}$ should also be related to the magnitude of the squared norm $\|\boldsymbol{E}\|^{2}$, which is much larger than those of the two derivatives. Additionally, for phantoms where the emissivity distribution is local and narrow in the flux-tube direction, the best value of $\gamma_{1}$ has tended to decrease. Based on these simulation results, the scans of $\gamma_{1}, \alpha$ and $\beta$ are made efficiently in narrowed ranges for the rest of analyses.

\section{[2] Regularization effects in reconstructed images}

The two derivative terms $\left\|\boldsymbol{D}_{1} \boldsymbol{E}\right\|^{2}$ and $\left\|\boldsymbol{D}_{2} \boldsymbol{E}\right\|^{2}$ give meaningful effects to the profiles of reconstructed images. In the simulations, the reconstruction is studied in changing the regularization term design.

Firstly, comparison is made in two cases of using only the Tikhonov term $\|\boldsymbol{T E}\|^{2}$ and using all three terms. Figures 7 and 8 show a result in the simulation for which the result in Fig. 6 was obtained. With similarly good fittings to the phantom projection as recognized in Fig. 7, the two methods give reconstructions with a remarkable difference as in Fig. 8(a) and (b), where both the phantom in Fig. 5 and the reconstructed images are raster-scanned and plotted in one dimension. In this plot of $E_{\mathrm{ph}, \mathrm{k}}$, the phantom profile is discontinuous in its existence intervals and oscillates like saw teeth in corresponding to the linear increase of emissivity with $L_{\mathrm{dp}}$. With the two derivative terms and the optimization with GCV, it is found that the reconstruction is much improved in giving a better recovery of the gradient of phantom along flux tubes and the noisy structure effectively diminished. The iterative procedure for non-negativity, with 16 iterations in this example, has worked well without producing any large residual. The iterative update of the operator $\mathbf{T}$, which has worked well also in case of the single term $\|\mathbf{T} \boldsymbol{E}\|^{2}$, can be a tool for eliminating the noisy negative values that appear in the original Tikhonov regularization [21, 22]. The tool is available wherever one has the linear regularization software.

In Fig. 9, the reconstructed images are illustrated for some of various regularization designs. The numerical sequences (red lines) in Figs. 8 (a) and (b) are displayed in Figs. 9 (a) and (d), now with cross sectional illustrations at different $L_{\mathrm{dp}}$. The image in Fig. 9 (b) is obtained by imposing a strong constraint that the objective emissivity is constant along the flux tubes, instead of adding the parallel derivative term $\left\|\boldsymbol{D}_{1} \boldsymbol{E}\right\|^{2}$. The reconstruction so- 
modified is made simply by decreasing the size of $\boldsymbol{E}$ to the number of flux tubes and summing the related row vectors of the projection matrix $\boldsymbol{L}$. Evidently, the flux constant constraint can never give good effects. In contrast, the addition of $\left\|\boldsymbol{D}_{2} \boldsymbol{E}\right\|^{2}$ term improves the reconstruction clearly as shown in Fig. 9 (c).

The reconstruction is further improved by adding the term $\left\|\boldsymbol{D}_{2} \boldsymbol{E}\right\|^{2}$ as seen in Fig. 9 (d), where the reconstruction error $\delta$ is decreased.

\section{[3] Regularization effects in numerical components}

The improved image displayed in Figs. 8(b) and 9(d) is examined on its spectral components, which are given a significant change by applying the two derivative terms.

In Figures 10, 11 and 12 are exhibited the singular value series, the Tikhonov window, the spectral coefficients and a couple of basis systems, which were used to calculate the image according to Eq. (5). The $\sigma_{\mathrm{m}}$ as well as $w_{m}$ gradually decrease with increasing $\mathrm{m}$. The spectral coefficients of $\boldsymbol{S}$ and $\boldsymbol{E}\left(\gamma_{1}\right)$ in Figs. 10(b) and (c) are compressed nicely in the region of low spatialfrequency (small number $m$ ), especially, at $m=1$. This compression is supported by the basis systems that are displayed in Figs. 11 and 12. The bases $\mathbf{R}^{-1} \mathbf{v}_{\mathrm{m}}$ for $\boldsymbol{E}\left(\gamma_{1}\right)$ change in profile with the increase of $m$ from smooth to random functions. In particular, the basis $\mathbf{R}^{-1} \mathbf{v}_{1}$ has a distribution that is similar to the emission region in shape, and thereby supports the strong spectral compression of $\boldsymbol{E}\left(\gamma_{1}\right)$ at $\mathrm{m}=1$ and the related smooth profile seen in Fig. 9(d). The great profile change of $\mathbf{R}^{-1} \mathbf{v}_{\mathrm{m}}$ in the interval of 1 $\leqq \mathrm{m} \leqq$ M suggests that the basis system can support various emissivity distributions, occasionally with fine structures. Contrarily, the regularization only with $\|\mathbf{T E}\|^{2}$ fails in generation of smooth bases for small $\mathrm{m}$ and, as a result, gives the unstable profile of emissivity as seen in Fig. 9(a). This notable improvement of basis system has been obtained by involving the derivative terms in regularization.

The above results have been obtained with the Titeration for non-negativity and with the GCVoptimization according to the previously described algorithm. In Fig. 9(b) and (d), the spectral coefficients are plotted in magnitude for graphic convenience. Calculation was carried out with the software Matlab.

\section{Application to impurity emissions in the LHD edge region}

Impurity emissions in the LHD experiments, CII $\left(1 \mathrm{~s}^{2} 2 \mathrm{~s} 2 \mathrm{p} 3 \mathrm{~s}{ }^{4} \mathrm{P}^{\mathrm{o}}-1 \mathrm{~s}^{2} 2 \mathrm{~s} 2 \mathrm{p} 3 \mathrm{p}{ }^{4} \mathrm{P}, 514 \mathrm{~nm}\right), \operatorname{CIV}\left(1 \mathrm{~s}^{2} 5 \mathrm{f}^{2} \mathrm{~F}^{\mathrm{o}}\right.$ $\left.1 \mathrm{~s}^{2} 6 \mathrm{~g}{ }^{2} \mathrm{G}, 1 \mathrm{~s}^{2} 5 \mathrm{~g}{ }^{2} \mathrm{G}-1 \mathrm{~s}^{2} 6 \mathrm{~h}{ }^{2} \mathrm{H}^{0}, 466 \mathrm{~nm}\right)$ are analyzed with the tomography method described above. Figure 13 shows CII and CIV projection images at the detector and the recovered ones in tomography. Here we have $n_{\mathrm{e}}=$
$2 \times 10^{19} \mathrm{~m}^{-3}$, NBI heating power of $13,14 \mathrm{MW}$, shot numbers 133323, 137177, $\mathrm{t}=4.75,4.30 \mathrm{sec}$, exposure time $=0.15,0.30 \mathrm{sec}$, gratings of 150,2400 grating $/ \mathrm{mm}$, respectively, for CII and CIV measurements $\left(\gamma_{1}=\right.$ $\left.2.6 \times 10^{-6}, 1.0 \times 10^{-6} ; \alpha=10^{-3}, \beta=10^{-6}\right)$. For the reasonably recovered projections, the reconstructed $3 \mathrm{D}$ images are shown in Fig. 14 at different distances from the detector, $L_{\mathrm{dp}}$. In reconstruction, the emissions are peaked at the left divertor leg of $L_{\mathrm{dp}}=5.5 \mathrm{~m}$ for both CII and CIV. This is considered due to strong plasma-wall interaction caused by divertor shaping at the top of the torus that terminates the divertor leg field lines with rather large incident angle. The emission of CIV is found to peak around the X-point and also distributed in poloidal direction with a certain modulation. On the other hand, there exists no clear peak in the CII emission except for the left leg. These differences between CII and CIV emission distributions are probably attributed to different ionization potentials, $24.4 \mathrm{eV}$ for CII and $64.7 \mathrm{eV}$ for CIV. That means CIV emission occur in higher temperature region.

Based on this experimental result, the following point may be noted for application and improvement of the tomography method for the edge plasma. In the edge region, strongly peaked emissions can exist especially near the divertor plates due to strong plasma-wall interaction, as observed also in the present analysis. In this case, reconstruction needs basic functions with high orders $m$ to reproduce such peaked distributions. The basic functions with higher $\mathrm{m}$, on the other hand, has potential to increase the spatial oscillation of emission by amplifying noise components, and thus tend to be suppressed by the window function. Therefore, the selection of regularization parameters that control smoothness of the solution should be made carefully by considering possible peaks appearing near plasma-facing components. In order to avoid any misleading reconstruction caused by the peaked distributions, separation of the control volume with possible peaked solution from the others in tomography analysis may be one solution.

\section{Summary}

A single field-of-view tomography method has been proposed for inverting the impurity emission distributions measured in magnetized edge plasma. Several regularization terms have been introduced taking into account the plasma transport characteristics in the magnetized plasma, in addition to the conventional Tikhonov regularization. The new scheme has been tested with the phantom data that varies the emissivity along field line direction. The effectiveness of the regularization terms has been confirmed in regard to the reconstruction error that can be reduced with the introduced regularization of 
derivative operators parallel and perpendicular to the magnetic field lines. In the test, the selection of regularization parameter values has also been discussed. It is found that the test image is effectively compressed to the Fourier-type components of low order, $\mathrm{m}=1$ and around $m=20$. The scheme has been applied to the experimental data in LHD. Different distributions are obtained for CII $\left(\mathrm{C}^{1+}\right)$ and $\mathrm{CIV}\left(\mathrm{C}^{3+}\right)$, which seem reasonable in terms of ionization potentials of each charge state and of the plasma-wall interaction. Special care should be taken for tomography analysis in the edge plasma, where strongly peaked emission can exist due to plasma-wall interaction, which may conflict with regularizations that usually promote smooth distribution of solutions.

\section{Acknowledgements}

One of the authors, N.I., is grateful to Prof. Yohsuke Hosoda for the fruitful discussions on the Cholesky decomposition in the tomography. The authors are also grateful to Prof. B.J. Peterson and Dr. H. Tsuchiya for the fruitful discussions. This work has been financially supported by Japan Society for the Promotion of Science Grant No. 16H04622, and NIFS budget code ULPP026.

\section{References}

${ }^{1}$ M. Kobayashi et al., Nucl. Fusion 55 (2015) 104021.

${ }^{2}$ N. Ohyabu et al., Nucl. Fusion 34 (1994) 387.

${ }^{3}$ Y. Feng et al., Nucl. Fusion 48 (2008) 024012.

${ }^{4}$ M. Kobayashi et al., Nucl. Fusion 53 (2013) 093032.
${ }^{5}$ M. Kobayashi et al., Nucl. Materials and Energy 12 (2017) 1043.

${ }^{6}$ T. Kobayashi et al., Plasma Fusion Res. 13 (2018) 3402030.

${ }^{7}$ S. Morita et al., Plasma Phys. Control. Fusion 56 (2014) 094007.

${ }^{8}$ M. Kobayashi et al., Rev. Sci. Instrum. 88 (2017) 033501.

${ }^{9}$ N. Iwama et al., Appl. Phys. Lett. 54 (1989) 502.

${ }^{10}$ N. Iwama: "Tomography for discharge plasmas," edited by K.H. Becker et al., AIP Press (New York, 1996), pp. 289-298.

${ }^{11}$ N. Terasaki, Y. Hosoda et al., Fusion Eng. and Design 34-35 (1997) 801.

${ }^{12}$ S. Ohdachi et al., Plasma Sci. and Tech. 8 (2006) 45.

${ }^{13}$ Dong Li et al., Rev. Sci. Instrum. 84 (2013) 083506.

${ }^{14}$ A. Wingen et al., J. Comp. Phys. 289 (2015) 83.

${ }^{15}$ J. Bielecki et al., Rev. Sci. Instrum. 86 (2015) 093505.

${ }^{16}$ M. Anton, H. Weisen et al., Plasma Phys. Control. Fusion 38 (1996) 1849.

${ }^{17}$ J. Mlynar et al., Fusion Sci. and Tech. 58 (2010) 733.

${ }^{18} \mathrm{M}$. Odstrcil, J. Mlynar et al., Nucl. Instrum. and Methods in Phys. Res. A 686 (2012) 156.

${ }^{19}$ J.M. Gao, Y. Liu et al., Rev. Sci. Instrum. 84 (2013) 093503.

${ }^{20}$ N. Iwama et al., J. Plasma Fusion Res. SERIES 8 (2009) 691.

${ }^{21}$ R. Sano et al., Rev. Sci. Instrum 87 (2016) 053502.

${ }^{22}$ R. Sano et al., Rev. Sci. Instrum. 87 (2016) 11D440.

${ }^{23}$ K. Ertl, W. von der Linden et al., Nucl. Fusion 36 (1996) 1477. 


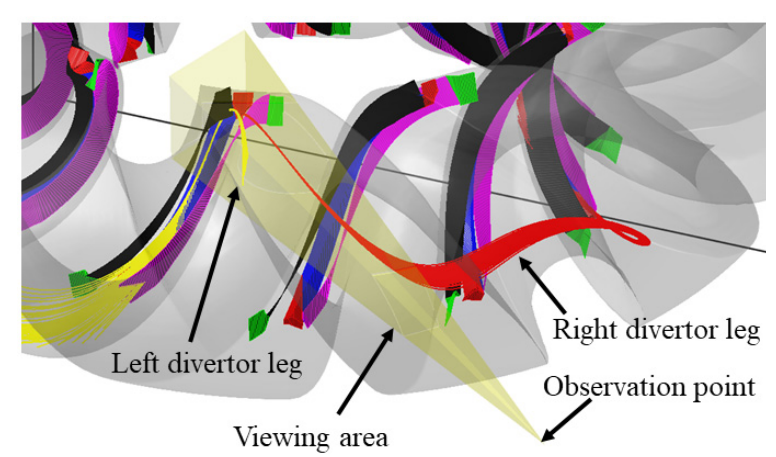

Fig.1 The viewing area of the spectrometer shaded by yellow (quadrangular pyramid). The divertor leg field lines are shown with yellow and red lines. The divertor plate arrays at inboard side are shown with black and pink surfaces. The vacuum vessel shape is shown with transparent gray color.

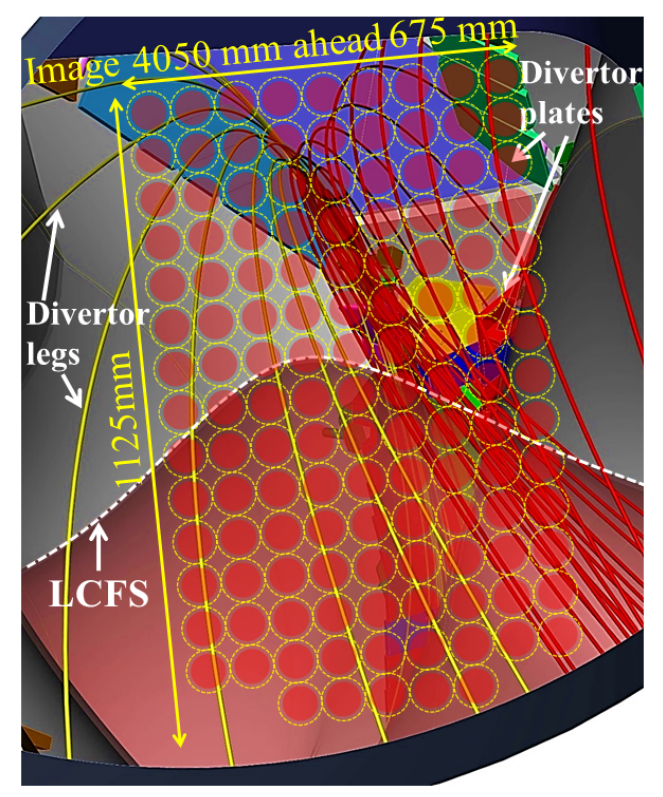

Fig.2 Field of view and fiber distribution for the twodimensional measurement. Yellow and red lines represent divertor legs, and dashed line indicates the LCFS.

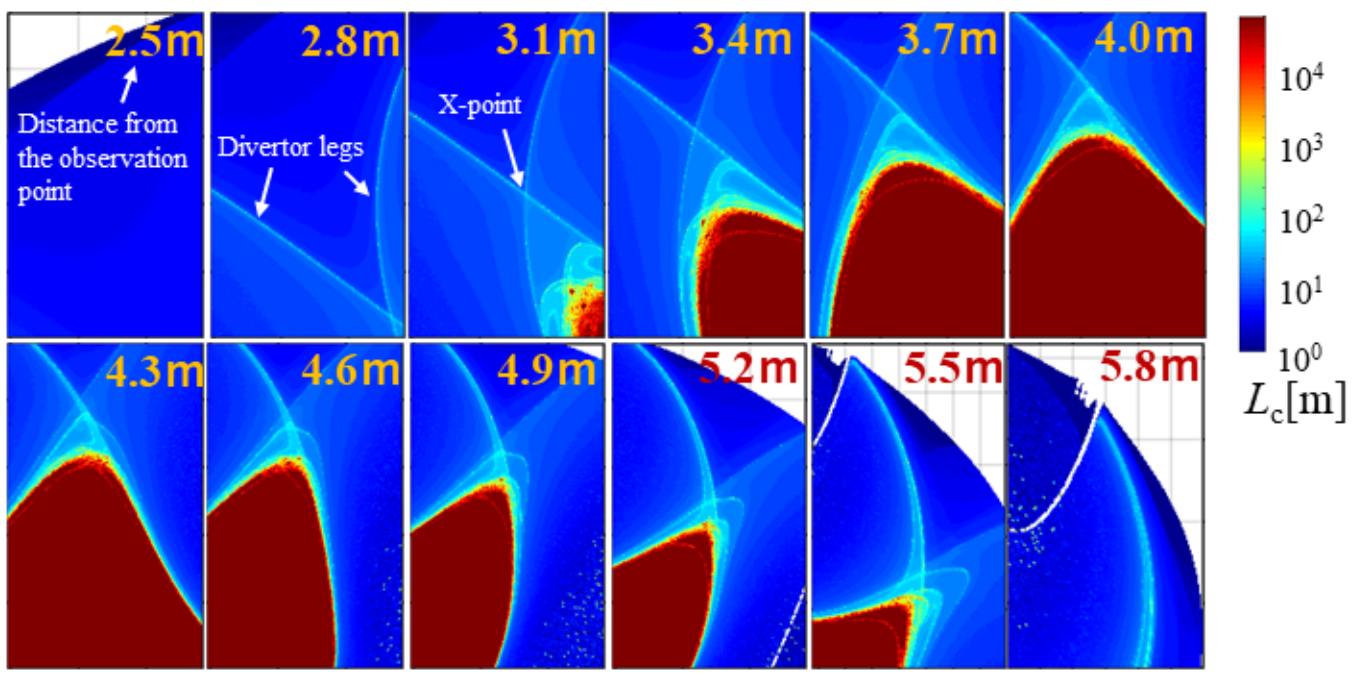

Fig.3 Connection length $\left(L_{\mathrm{C}}\right)$ distribution in cross sections perpendicular to LOS. Length in each panel indicates distance from the observation point. Two divertor legs are visible with thin lines of blue color, and they cross each other at X-point. 

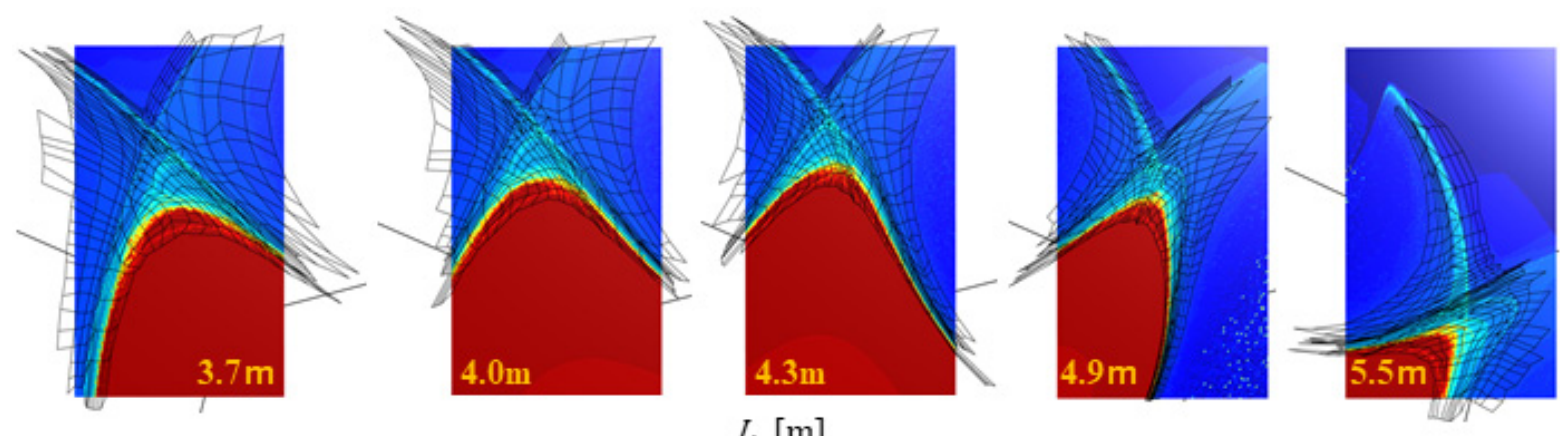

$$
L_{\mathrm{c}}[\mathrm{m}]
$$

$\begin{array}{lllll}10^{4} & 10^{3} & 10^{2} & 10^{1} & 10^{0}\end{array}$

Fig.4 Field-aligned 3D grid constructed for tomography: cross sectional illustrations with the label $L_{d p}$. Vertices are aligned along the magnetic field lines.

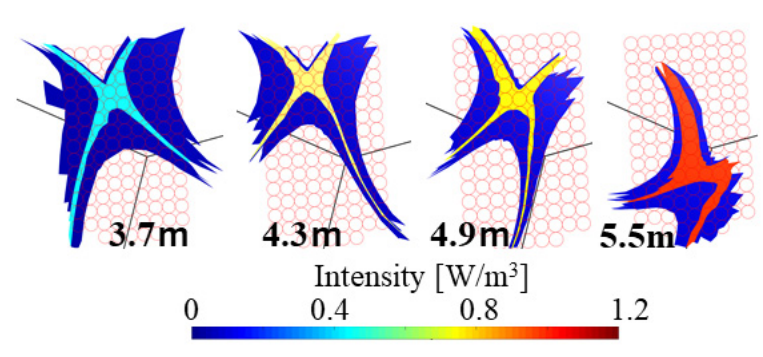

Fig.5 3D profile of a phantom. The variation of emissivity along LOS is shown with cross-sectional illustrations at different $L_{d p}$. Red circles in each panel indicate the fiber positions.

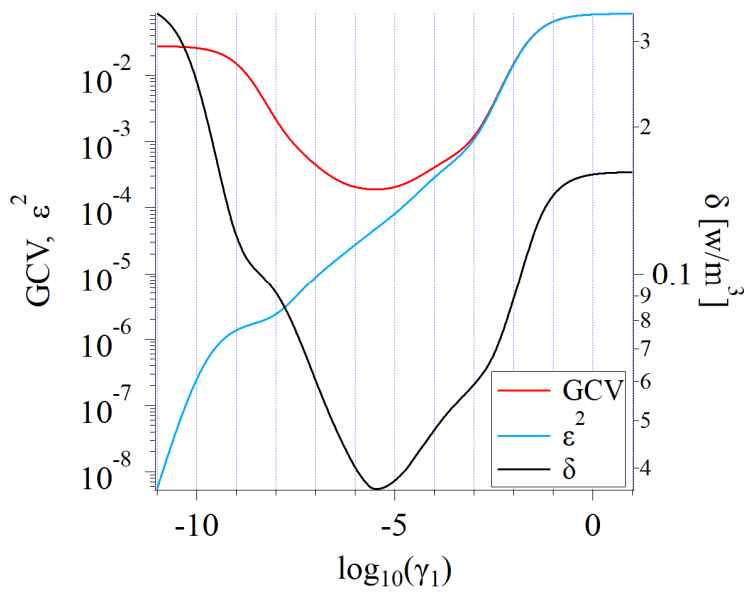

Fig. 6 Behaviors of GCV, $\varepsilon^{2}$ and $\delta$ when the parameter $\gamma_{1}$ is changed for fixed values of $\alpha=10^{-2}$ and $\beta=10^{-6}$. 


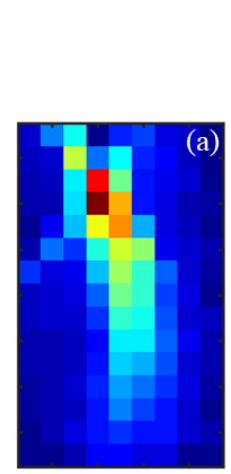

Projection image
Tikhonov term

$+1^{\text {st }}$ differentiation term

Tikhonov term $+2^{\text {nd }}$ differentiation term

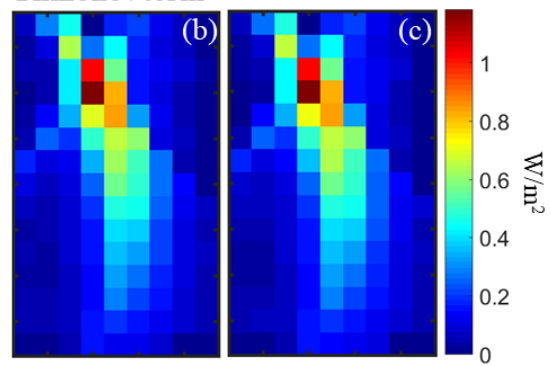

Reprojection image

Fig. 7 (a) Projection image of the phantom with no noise, those recovered with (b) the Tikhonov term only and (c) all terms.

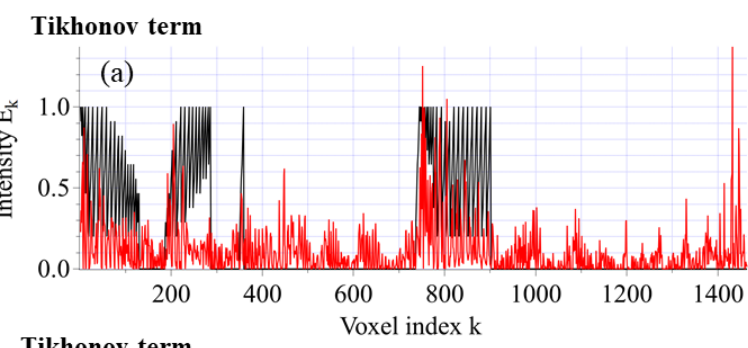

Tikhonov term

$+1^{\text {st }}$ differentiation term

$+2^{\text {nd }}$ differentiation term

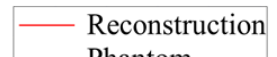

(b)

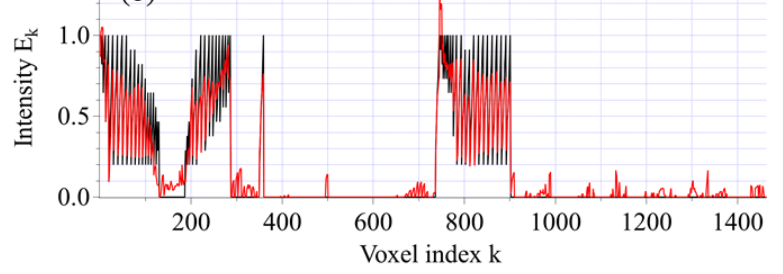

Fig. 8 1D display of the phantom and the images reconstructed with (a) the Tikhonov term only and (b) all terms. 1464 voxels are indexed in sequence by raster scan along the LOS of fibers.

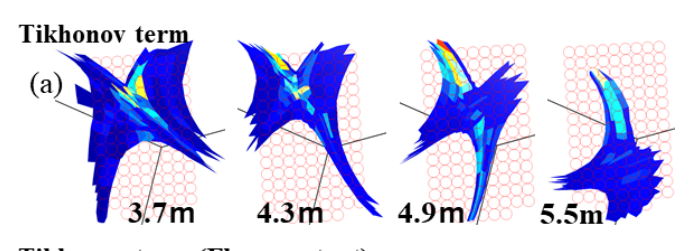

Tikhonov term (Flux constant)

(b)
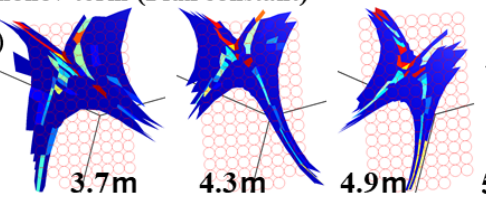

$4.3 \mathrm{~m}$

$4.9 \mathrm{~m} /$

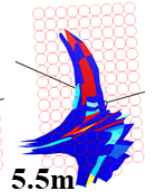

Tikhonov term $+2^{\text {nd }}$ differentiation term

(c)

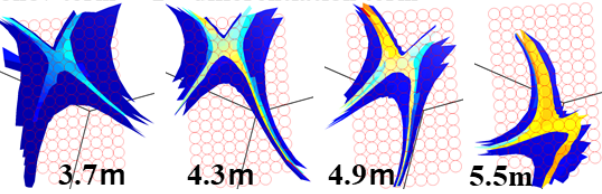

Tikhonov term $+1^{\text {st }}$ differentiation term $+2^{\text {nd }}$ differentiation term

(d)

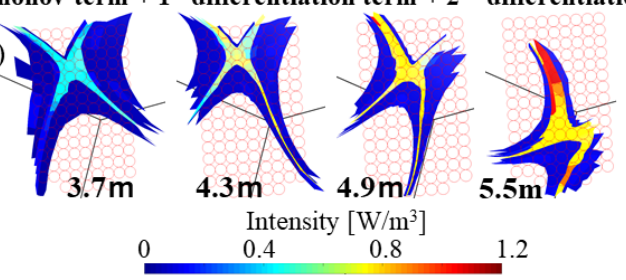

Fig. 9 Reconstructed images for different designs of regularization: (a) $\|\boldsymbol{T} \boldsymbol{E}\|^{2}$ only $\left(\delta=1.8 \times 10^{-1}\right)$, (b) $\|\boldsymbol{T E}\|^{2}$ with the constraint of flux constant $(\delta=$ $\left.3.0 \times 10^{-1}\right)$, (c) $\|\boldsymbol{T} \boldsymbol{E}\|^{2}$ and $\left\|\boldsymbol{D}_{1} \boldsymbol{E}\right\|^{2}\left(\delta=7.1 \times 10^{-2}\right)$, and (d) all terms $\left(\delta=3.6 \times 10^{-2}\right)$. 

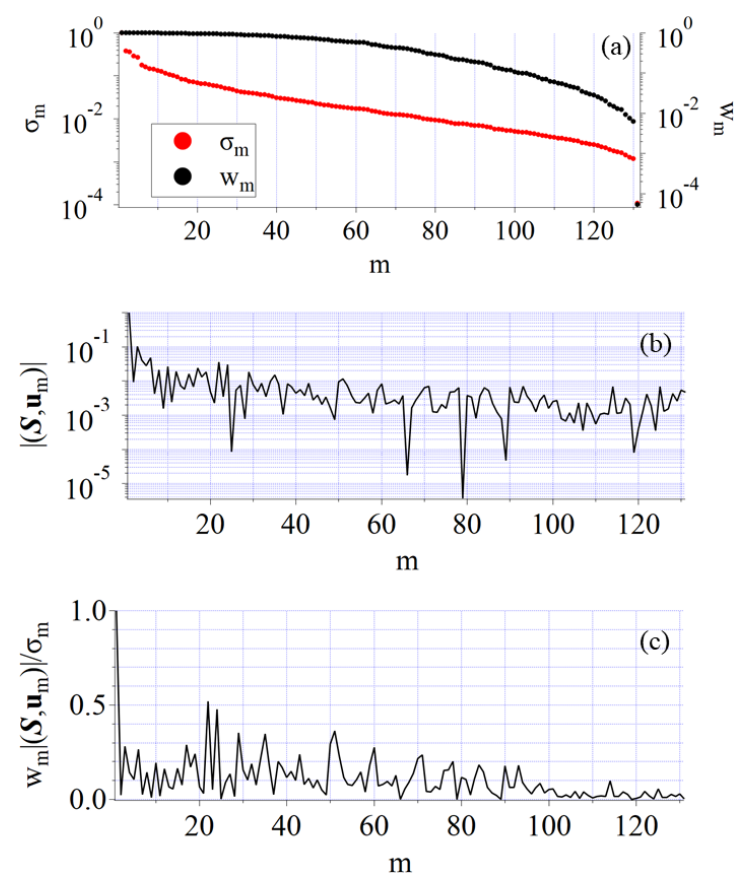

Fig. 10 (a) Singular value series $\sigma_{\mathrm{m}}$ normalized with $\sigma_{1}$ and the window function $\mathrm{w}_{\mathrm{m}}$, (b) the spectrum of data $S$, and (c) the normalized spectrum of the image $\boldsymbol{E}\left(\gamma_{1}\right)$ obtained with all regularization terms $\left(\gamma_{1}=10^{-5}\right.$, $\alpha=10^{-2}, \beta=10^{-6}$ ).
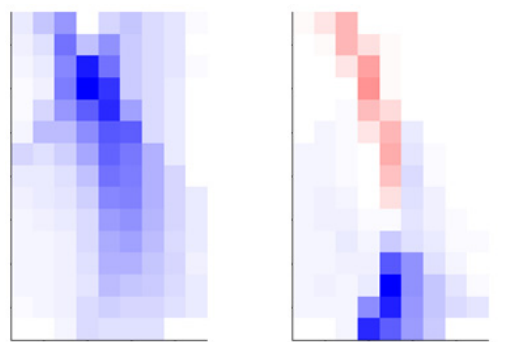

$\mathrm{m}=3$

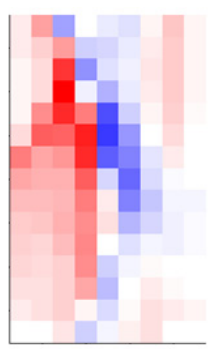

$\mathrm{m}=1$

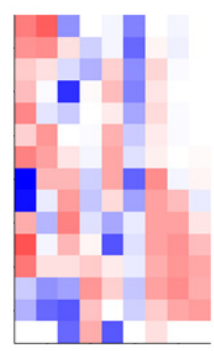

$\mathrm{m}=20$

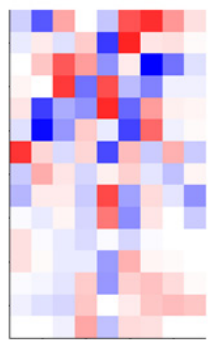

$\mathrm{m}=30$

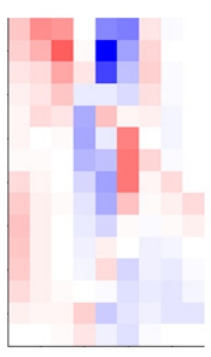

$\mathrm{m}=10$ (a)

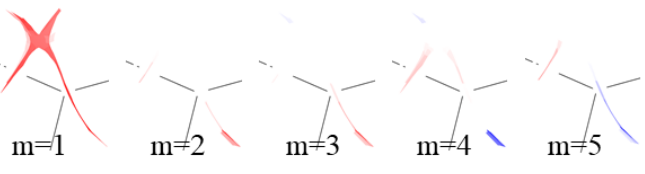

1 $\mathrm{m}=22 \backslash \mathrm{m}=24 、 \mathrm{~m}=29 \quad \mathrm{~m}=35 \backslash \mathrm{m}=51 \backslash$ $\begin{array}{lllll}1 & 0.5 & 0 & -0.5 & -1\end{array}$

(b)

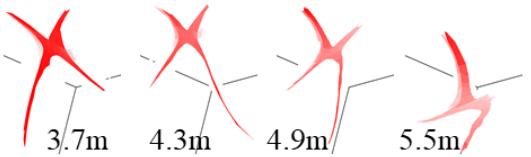

Fig.12 Bases $\boldsymbol{R}^{-1} \boldsymbol{v}_{\mathrm{m}}$ for the spectral decomposition in Fig. 10(c): (a) Cross sectional display for various $m\left(L_{\mathrm{dp}}=4.3[\mathrm{~m}]\right)$, where each basis is normalized with its maximum value. (b) Cross sections of $\boldsymbol{R}^{-1} \boldsymbol{v}_{1}$ at different $L_{d p}$. The inversion in color-bar is in correspondence with the sign inversion of spectrum in Fig. 10 (c).

$\begin{array}{lllll}1 & 0.5 & 0 & -0.5 & -1\end{array}$

Fig. 11 Bases $\boldsymbol{u}_{\mathrm{m}}$ for the spectral decomposition in Fig. 10(b). 
CII $n_{\mathrm{e}}=2.2 \times 10^{19} \mathrm{~m}^{-3}$ shot133323 $\mathrm{t}=4.75 \mathrm{~s}$

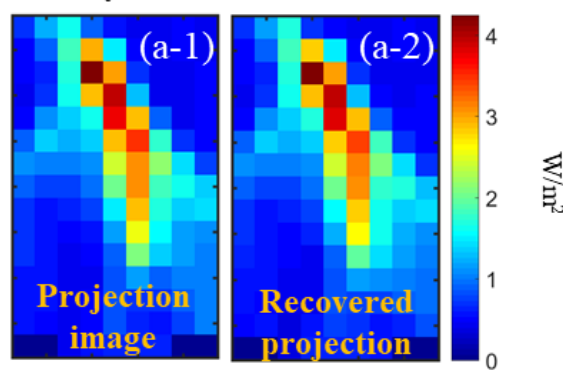

CIV $n_{\mathrm{e}}=2.1 \times 10^{19} \mathrm{~m}^{-3}$ shot $137177 \mathrm{t}=4.30 \mathrm{~s}$
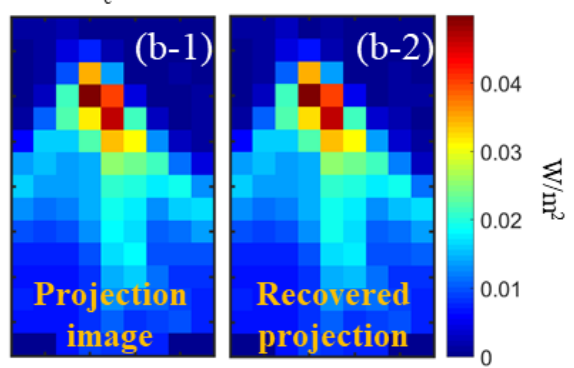

Fig.13 (a) Projection data in LHD experiment and (b)

the recovered projections for CII and CIV.

(a) CII $n_{\mathrm{e}}=2.2 \times 10^{19} \mathrm{~m}^{-3} \operatorname{shot} 133323 \mathrm{t}=4.75 \mathrm{~s} \quad \mathrm{~W} / \mathrm{m}^{3}$

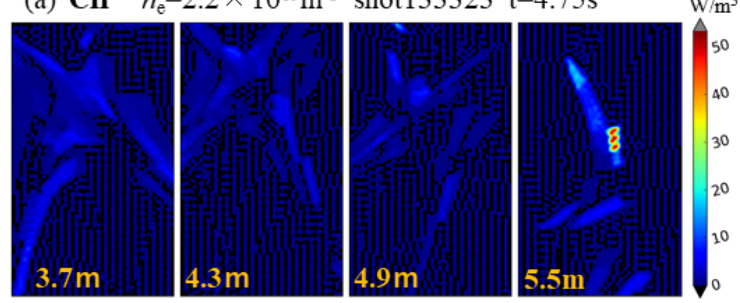

(b) CIV $n_{\mathrm{e}}=2.1 \times 10^{19} \mathrm{~m}^{-3} \operatorname{shot} 137177 \mathrm{t}=4.30 \mathrm{~s}$

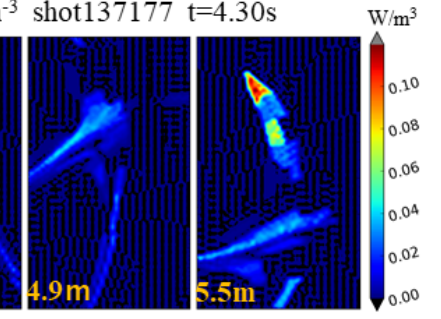

Fig. 14 Reconstructed images of (a) CII and (b) CIV in cross sectional display at different $L_{\mathrm{dp}}$. 